\title{
Treatment options for refractory/relapsed multiple myeloma: an updated evidence synthesis by network meta-analysis
}

This article was published in the following Dove Press journal: Cancer Management and Research

\author{
Xian-Wu Luo' \\ Xue-Qing $\mathrm{Du}^{2}$ \\ Jie-Li Li ${ }^{2}$ \\ Xiao-Ping $\operatorname{Liu}^{3,4}$ \\ Xiang-Yu Meng 3 ,4
}

'Department of Health Management, School of Health Sciences, Wuhan University, ${ }^{2}$ Department of Hematology, Zhongnan Hospital of Wuhan University, ${ }^{3}$ Department of Evidence-Based Medicine and Clinical Epidemiology, Second Clinical School, Wuhan University, ${ }^{4}$ Center for Evidence-based and Translational Medicine, Zhongnan Hospital of Wuhan University, Wuhan, China
Correspondence: Xiang-Yu Meng Department of Evidence-Based Medicine and Clinical Epidemiology, Second Clinical School, Wuhan University, 169 Donghu Road, Wuhan 43007I, China

$\mathrm{Tel} / \mathrm{Fax}+862767812817$

Email mengxy_whu@।63.com
Background: The refractory/relapsed multiple myeloma (RRMM) remains a big clinical challenge, due to its biological and clinical complexity. Leading hematologists have performed many randomized controlled trials (RCTs) worldwide, and their findings were summarized in a recently published network meta-analysis (NMA) but with certain limitations.

Materials and methods: We performed an updated NMA of RCTs related to RRMM treatment, focusing on efficacy measures including the nonresponse rate (NRR), time to progression (TTP), progression-free survival (PFS), and overall survival (OS). The PubMed database was searched. We extended the literature search strategy of a previous NMA to June 30, 2017 and included additional primary RCTs. The surface under the cumulative ranking curve (SUCRA) was calculated to rank the regimens. A weighted-average method was used to rank the regimens by summarizing SUCRAs across different outcome measures.

Results: Finally, a total of 24 RCTs were included in this updated NMA. According to the result, the combination of daratumumab, lenalidomide, and dexamethasone showed better efficacy than other regimens in terms of NRR, TTP, and PFS (NRR: odds ratio [OR] $=0.046$, $95 \%$ credible interval $[\mathrm{CrI}]=[0.024,0.085]$; TTP: hazard ratio $[\mathrm{HR}]=0.14,95 \% \mathrm{CrI}=[0.092$, $0.2]$; PFS: $\mathrm{HR}=0.12,95 \% \mathrm{CrI}=[0.077,0.18]$, compared with dexamethasone singlet). The combination of ixazomib, lenalidomide, and dexamethasone showed better efficacy than other regimens in terms of $\mathrm{OS}$ ( $\mathrm{HR}=0.30,95 \% \mathrm{CrI}=[0.17,0.54]$, compared with dexamethasone). The combination of daratumumab, lenalidomide, and dexamethasone ranked first in terms of overall efficacy (weighted average of SUCRAs $=0.920$ ).

Conclusion: The combination of daratumumab, lenalidomide, and dexamethasone may currently be the most effective regimen in the population of RRMM patients. Triplet regimens containing daratumumab, ixazomib, carfilzomib, or elotumumab plus lenalidomide and dexamethasone can be recommended as first-line therapies for RRMM patients.

Keywords: multiple myeloma, refractory/relapsed, treatment regimens, efficacy, network meta-analysis

\section{Introduction}

Since the early 2000s, the introduction of newly developed agents has dramatically improved the outcome of patients with multiple myeloma. However, the refractory/ relapsed multiple myeloma (RRMM) remains a big challenge, due to its biological and clinical complexity. In an attempt to find ways to conquer RRMM, world's leading hematologists have performed many randomized controlled trials (RCTs) worldwide. Recently, two research groups have tried to synthesize the results of these trials by network meta-analysis (NMA), a statistical analysis method by which a thorough 
comparison and ranking of all included treatment options is made possible. ${ }^{1,2}$ However, the two previous NMAs have certain limitations. To name a few, in the NMA by van BeurdenTan et al, ${ }^{1}$ important outcomes such as overall survival (OS) were left uninvestigated and the ranking information was not provided; and in the other NMA by Botta et al, ${ }^{2}$ regimens were grouped into categories before comparison, some of which seem inappropriate and may cause bias and confusion. Besides, we found that several new RCTs and updated trial reports are available for a new NMA. Considering the above, we performed the present study to update the evidence and improve its quality using traditional NMA and a self-designed weighted average method to rank the regimens by summarizing results across various efficacy outcome measures.

\section{Materials and methods}

\section{Outcome measures}

This updated NMA focuses on early and long-term efficacy outcomes, including the nonresponse rate (NRR), time to progression (TTP), progression-free survival (PFS) and OS. Response rate was considered instead of NRR because its outcome event, that is, the absence of objective response to treatment, a negative indicator of treatment efficacy and prognosis, is similar to events of other outcome measures, which allows ranking by summarizing results across all these outcome measures.

\section{Literature search and study selection}

The PubMed database was searched. We adopted the literature search strategy described by Botta et al. ${ }^{2}$ In brief, all possible combinations of the following search terms were used for searching RCTs concerning RRMM patients: "multiple myeloma," "relapse," "refractory," "randomized," "management," "regimen," and "therapy." No specific filters were used during the search. The time range of the search was between the January 1, 2000 and June 30, 2017.

The following predefined eligibility criteria were used for the study selection: 1) the study should be an RCT; 2) the subjects should be RRMM patients; 3) at least two different regimens were compared in the study, except for those comparing different dosing schemes or modes of administrations; 4) data for at least one of the outcome measures were available. Studies that did not match any of the above criteria were excluded.

Two authors (X.W.L. and X.Q.D.) independently performed the literature search and study selection and discussed with the third author (X.M.) to resolve any discrepancies.

\section{Data extraction and statistical analysis}

Two authors (X.W.L. and X.Q.D) independently reviewed the reports. Supplementary materials of the RCTs were included, and the following information was extracted from each study report: name of the first author, year of publication, trial identifications, treatment regimens used for the experimental and control arms, total number of patients, outcome measures investigated, and data for the calculation of effect size for each outcome.

Before NMA, the logarithmic odds ratio (OR) and its standard error were calculated for NRR, and the logarithmic hazard ratio (HR) and its standard error for time-to-event outcomes were original data inputs. Fixed effects Bayesian NMAs were conducted, and forest plots were generated with results shown as the HR and corresponding 95\% credible interval $(95 \% \mathrm{CrI})$. To include all treatments within one network, bortezomib with or without dexamethasone was considered identical and labeled as "bortezomib \pm dexamethasone" and thalidomide with or without dexamethasone was considered identical and labeled as "thalidomide \pm dexamethasone" as described in van Beurden-Tan et al. ${ }^{1}$ The dexamethasone monotherapy was set as the common reference regimen. To rank all regimens, the surface under the cumulative ranking curve (SUCRA) was calculated for each outcome as described in Salanti et al ${ }^{3}$ for a given regimen. A larger SUCRA score indicated better efficacy in terms of a specific outcome measure. For the final ranking regarding the overall efficacy, an arithmetic weighted average of SUCRA scores across NRR, PFS, and OS was calculated. A $20 \%, 35 \%$, and $45 \%$ weight was given to the NRR, PFS, and $O S$, respectively. These weights were given in accordance with the importance of the outcome measures. We considered the OS the most important, PFS as the surrogate of OS the second most important, and NRR that reflects the early efficacy the least important. There was only one exception, oblimersen plus dexamethasone, for which data on PFS and OS were not available, thus an $80 \%$ weight was attributed to TTP. A larger weighted average of SUCRAs indicated a higher rank in terms of overall efficacy. The R software version 3.1.2 and the gemtc package were used to perform all the statistical analyses.

\section{Results}

\section{Basic information of included RCTs}

After literature search and study selection, a total of 29 trial reports published between 2005 and 2017 were considered eligible to be included in the NMA. As a result, 24 
independent studies, with a total of 10,853 subjects and 21 different regimens were included in the present study. Compared with the inclusion results by Botta et al, ${ }^{2}$ an additional seven references ${ }^{4-10}$ were included, among which four were newly identified studies ${ }^{4,5,9,10}$ and three were updated study results. ${ }^{6-8}$ The basic information of the included references has been summarized in Table 1.

\section{NMA results}

According to the results of NMAs, the combination of daratumumab, lenalidomide, and dexamethasone was the most effective therapy in terms of NRR, TTP, and PFS (NRR: $\mathrm{OR}=0.046,95 \% \mathrm{CrI}=[0.024,0.085]$; TP: $\mathrm{HR}=0.14,95 \%$ $\mathrm{CrI}=[0.092,0.2]$; PFS: HR $=0.12,95 \% \mathrm{CrI}=[0.077,0.18]$, compared with dexamethasone singlet; Figure 1A-C), and the combination of ixazomib, lenalidomide, and dexamethasone is the most effective one in terms of $\mathrm{OS}$ ( $\mathrm{HR}=0.12,95 \% \mathrm{CrI}$ $=[0.17,0.54]$; Figure 1D).

\section{Ranking of regimens by SUCRA}

As shown in Table 2, the ranking by SUCRA scores for each efficacy outcome was generally consistent with NMA findings. The combination of daratumumab, lenalidomide, and dexamethasone ranked first for NRR (SUCRA $=0.984$ ), TTP (SUCRA $=0.988$ ), and PFS (SUCRA $=0.999$ ). The combination of ixazomib, lenalidomide, and dexamethasone ranked first for OS (SUCRA $=0.972$ ).

In terms of overall efficacy measured by the weighted average of SUCRAs, the combination of daratumumab, lenalidomide, and dexamethasone ranked on top (weighted average $=0.920$ ), followed by the combination of ixazomib, lenalidomide, and dexamethasone ( weighted average $=0.907$ ).

\section{Discussion}

Regardless of recent progress in management, RRMM is still an incurable disease, which has been attracting substantial research attention. In the past decades, a large number of

Table I Basic information of included RCT reports

\begin{tabular}{|c|c|c|c|c|c|c|}
\hline Authors & Year & Trial Identification & Arm I & Arm 2 & $\mathbf{N}$ & Outcome \\
\hline Richardson et al" & 2005 & APEX & BOR & DEX & 627 & NRR, TTP, OS \\
\hline Richardson et al ${ }^{12}$ & 2007 & APEX & BOR & DEX & 627 & NRR, TTP, OS \\
\hline Dimopoulos et $\mathrm{al}^{13}$ & 2007 & MM-0I0 & LEN + DEX & DEX & 351 & NRR, TTP, OS \\
\hline Orlowski et al ${ }^{14}$ & 2007 & DOXIL-MMY-300I & BOR + pIDOX & $B O R+D E X$ & 646 & NRR, TTP, PFS, OS \\
\hline Weber et al ${ }^{15}$ & 2007 & MM-009 & LEN + DEX & DEX & 353 & NRR, TTP, OS \\
\hline Chanan-Khan et al ${ }^{16}$ & 2009 & GMY302 & $O B L+D E X$ & DEX & 224 & NRR, TTP \\
\hline Dimopoulos et al ${ }^{17}$ & 2009 & MM-009 \& MM-0I0 & LEN + DEX & DEX & 704 & PFS, OS \\
\hline Garderet et $\mathrm{al}^{18}$ & 2012 & MMVAR/IFM 2005-04 & $\mathrm{BOR}+\mathrm{THA}+\mathrm{DEX}$ & THA + DEX & 244 & NRR, TTP, PFS, OS \\
\hline Hjorth et al ${ }^{19}$ & 2012 & NCT006025II & $\mathrm{THA}+\mathrm{DEX}$ & $\mathrm{BOR}+\mathrm{DEX}$ & $|3|$ & NRR, PFS, OS \\
\hline Kropff et a ${ }^{20}$ & 2012 & OPTIMUM & THA & DEX & 499 & NRR, TTP, PFS, OS \\
\hline Dimopoulos et $\mathrm{a}^{21}$ & 2013 & VANTAGE 088 & VOR + BOR & BOR & 635 & NRR, TTP, PFS, OS \\
\hline San-Miguel et al ${ }^{4}$ & 2013 & MM-003 & POM + DEX & DEX & 455 & NRR, TTP, PFS, OS \\
\hline White et al..$^{22}$ & 2013 & AMBER & $\mathrm{BEV}+\mathrm{BOR}$ & BOR & 102 & NRR, PFS, OS \\
\hline Morgan et al ${ }^{6}$ & 2014 & MM-003 & POM + DEX & DEX & 455 & OS \\
\hline Richardson et al ${ }^{5}$ & 2014 & MM-002 & $P O M+D E X$ & POM & 221 & NRR, PFS, OS \\
\hline San-Miguel et al ${ }^{23}$ & 2014 & PANORAMAI & $\mathrm{PAN}+\mathrm{BOR}+\mathrm{DEX}$ & $B O R+D E X$ & 768 & NRR, TTP, PFS, OS \\
\hline Lonial et $\mathrm{al}^{24}$ & 2015 & ELOQUENT-2 & ELO + LEN + DEX & LEN + DEX & 646 & NRR, PFS \\
\hline Orlowski et a ${ }^{25}$ & 2015 & NCT0040I843 & $\mathrm{SIL}+\mathrm{BOR}$ & BOR & 268 & NRR, TTP, PFS, OS \\
\hline Stewart et $\mathrm{al}^{26}$ & 2015 & ASPIRE & CAR + LEN + DEX & LEN + DEX & 792 & NRR, PFS, OS \\
\hline Dimopoulos et $\mathrm{al}^{27}$ & 2016 & POLLUX & DAR + LEN + DEX & LEN + DEX & 557 & NRR, TTP, PFS, OS \\
\hline Dimopoulos et $\mathrm{al}^{28}$ & 2016 & ENDEAVOR & CAR + DEX & $\mathrm{BOR}+\mathrm{DEX}$ & 929 & NRR, PFS, OS \\
\hline Jakubowiak et $\mathrm{al}^{29}$ & 2016 & NCTOI478048 & $\mathrm{ELO}+\mathrm{BOR}+\mathrm{DEX}$ & $B O R+D E X$ & 152 & NRR, PFS, OS \\
\hline Moreau et $\mathrm{a}^{30}$ & 2016 & TOURMALINE-MMI & IXA + LEN + DEX & LEN + DEX & 722 & NRR, TTP, PFS \\
\hline Orlowski et $\mathrm{a}^{31}$ & 2016 & DOXIL-MMY-300I & BOR + pIDOX & $B O R+D E X$ & 646 & OS \\
\hline Palumbo et $\mathrm{al}^{32}$ & 2016 & CASTOR & $\mathrm{DAR}+\mathrm{BOR}+\mathrm{DEX}$ & $\mathrm{BOR}+\mathrm{DEX}$ & 474 & NRR, TTP, PFS, OS \\
\hline San-Miguel et al ${ }^{7}$ & 2016 & PANORAMA I & $\mathrm{PAN}+\mathrm{BOR}+\mathrm{DEX}$ & $B O R+D E X$ & 768 & OS \\
\hline Dimopoulos et $\mathrm{al}^{8}$ & 2017 & ELOQUENT-2 & ELO + LEN + DEX & LEN + DEX & 646 & PFS, OS \\
\hline Hou et $\mathrm{al}^{10}$ & 2017 & NCT0I564537 & IXA + LEN + DEX & LEN + DEX & 115 & NRR, TTP, PFS, OS \\
\hline Hajek et al ${ }^{9}$ & 2017 & FOCUS & CAR & DEX & 315 & NRR, PFS, OS \\
\hline
\end{tabular}

Abbreviations: BOR, bortezomib; BEV, bevacizumab; CAR, carfilzomib; DAR, daratumumab; DEX, dexamethasone; pIDOX, Pegylated liposomal doxorubicin; ELO, elotumumab; IXA, ixazomib; LEN, lenalidomide; OBL, oblimersen; POM, pomalidomide; SIL, siltuximab; THA, thalidomide; NRR, non-response rate; PAN, panobinostat; PFS, progression-free survival; TTP, time to progression; OS, overall survival; $N$, number of patients; VOR, vorinostat. 


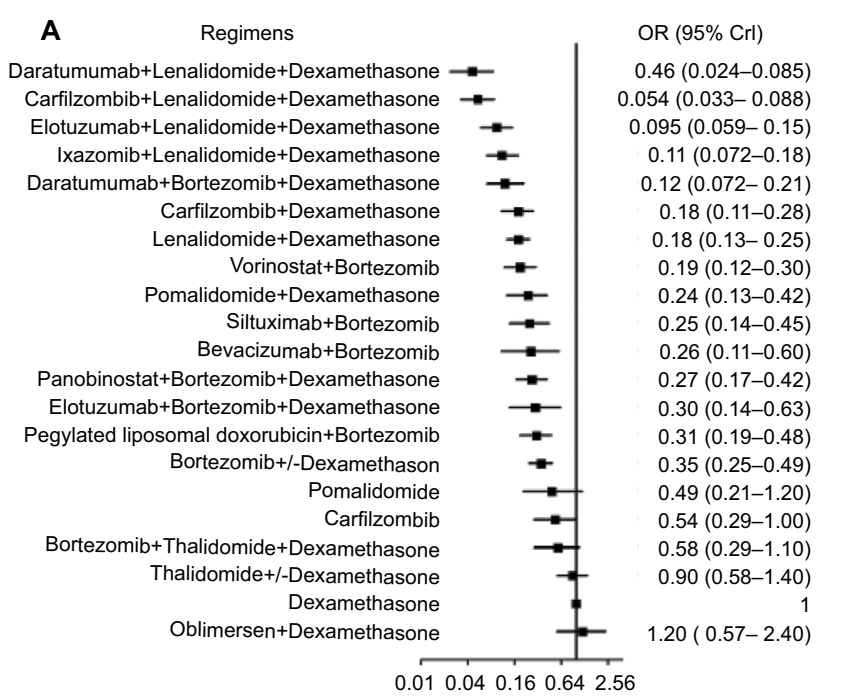

C

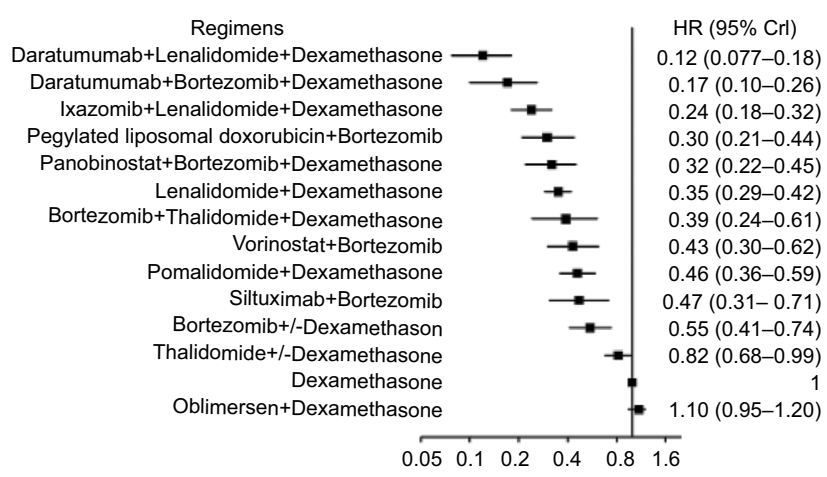

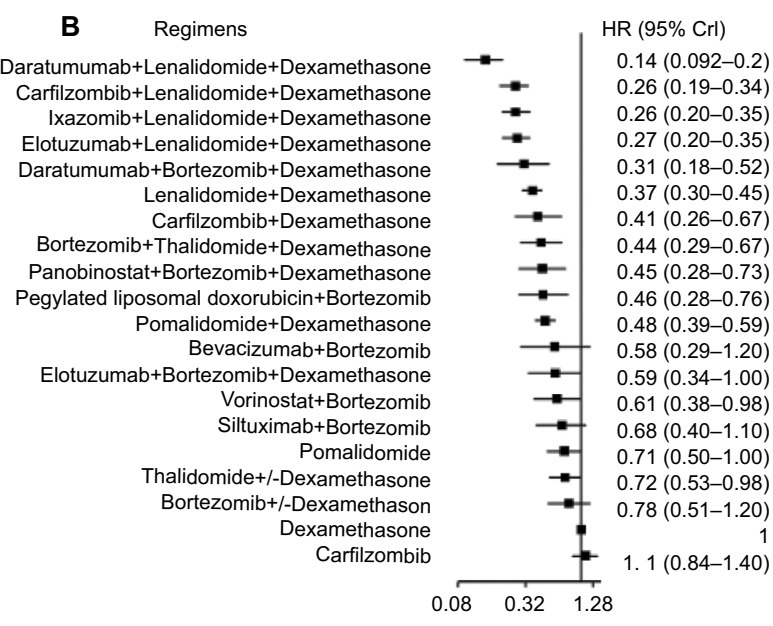

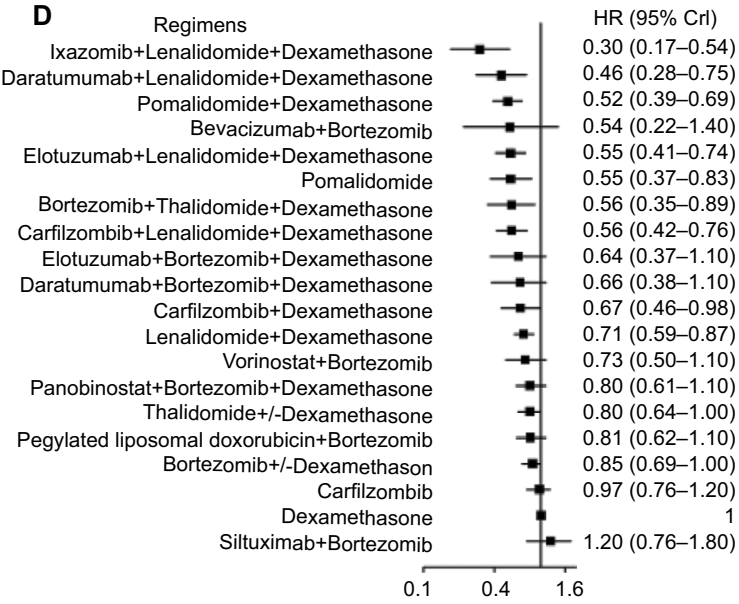

Figure I Forest plots presenting results of network meta-analysis: (A) nonresponse rate, (B) progression-free survival, (C) time to progression, (D) overall survival. Abbreviations: OR, odds ratio; $\mathrm{Crl}$, credible interval; $\mathrm{HR}$, hazard ratio.

RCTs concerning RRMM treatment were published, in which dozens of novel therapeutic regimens were tested and examined. Efforts were made to quantitatively summarize the evidence from RCTs by performing NMA, in order to provide useful and important information for clinical decision-making at minimal cost. However, flawed analyses may result in misleading conclusions. On the other hand, updates of NMAs on a timely basis are the key to keep the evidence "alive." The present study included the most recently published trials and updates of previous trial reports, thoroughly investigated the efficacy profiles of 21 treatment options, and avoided certain limitations of previous NMAs mentioned above.
According to our results, the combination of daratumumab, lenalidomide, and dexamethasone was most effective in terms of NRR, TTP, and PFS. However, in terms of OS, it was less effective compared with the combination of ixazomib, lenalidomide, and dexamethasone. Ranking by overall efficacy showed that the daratumumab, lenalidomide, and dexamethasone triplet regimen had better performance than other regimens. Interestingly, we noted that the 21 regimens investigated in this NMA can be categorized into three subgroups based on the weighted average of SUCRA scores. The first subgroup includes four regimens $(19.0 \%$, weighted average from $\sim 0.8$ to $\sim 0.9$ ), which are the most 
Table 2 Included regimens, SUCRAs, weighted averages of SUCRAs across NRR, TTP, and OS, and ranks in terms of overall efficacy

\begin{tabular}{|c|c|c|c|c|c|c|}
\hline Regimens & NRR & TTP & PFS & os & $\begin{array}{l}\text { Weighted } \\
\text { average }\end{array}$ & Rank \\
\hline Daratumumab + lenalidomide + dexamethasone & 0.984 & 0.988 & 0.999 & 0.829 & 0.920 & I \\
\hline Ixazomib + lenalidomide + dexamethasone & 0.828 & 0.828 & 0.869 & 0.972 & 0.907 & 2 \\
\hline Carfilzomib + lenalidomide + dexamethasone & 0.964 & NA & 0.887 & 0.697 & 0.817 & 3 \\
\hline Elotuzumab + lenalidomide + dexamethasone & 0.873 & NA & 0.855 & 0.72 & 0.798 & 4 \\
\hline Daratumumab + bortezomib + dexamethasone & 0.813 & 0.927 & 0.812 & 0.539 & 0.689 & 5 \\
\hline Pomalidomide + dexamethasone & 0.539 & 0.393 & 0.512 & 0.771 & 0.634 & 6 \\
\hline Carfilzomib + dexamethasone & 0.683 & NA & 0.638 & 0.527 & 0.597 & 7 \\
\hline Lenalidomide + dexamethasone & 0.66 & 0.609 & 0.685 & 0.447 & 0.572 & 8 \\
\hline Bortezomib + thalidomide + dexamethasone & 0.202 & 0.531 & 0.581 & 0.689 & 0.554 & 9 \\
\hline Bevacizumab + bortezomib & 0.5 & NA & 0.38 & 0.656 & 0.528 & 10 \\
\hline Elotuzumab + bortezomib + dexamethasone & 0.429 & NA & 0.358 & 0.564 & 0.465 & II \\
\hline Pomalidomide monotherapy & 0.252 & NA & 0.25 & 0.698 & 0.452 & 12 \\
\hline Vorinostat + bortezomib & 0.661 & 0.446 & 0.342 & 0.433 & 0.447 & 13 \\
\hline Panobinostat + bortezomib + dexamethasone & 0.472 & 0.678 & 0.566 & 0.327 & 0.43965 & 14 \\
\hline Pegylated liposomal doxorubicin + bortezomib & 0.406 & 0.715 & 0.55 & 0.305 & 0.41095 & 15 \\
\hline Thalidomide \pm dexamethasone & 0.083 & 0.153 & 0.221 & 0.325 & 0.2402 & 16 \\
\hline Bortezomib \pm dexamethason & 0.329 & 0.263 & 0.149 & 0.235 & 0.2237 & 17 \\
\hline Siltuximab + bortezomib & 0.511 & 0.391 & 0.259 & 0.048 & 0.21445 & 18 \\
\hline Carfilzomib monotherapy & 0.214 & NA & 0.029 & 0.132 & 0.11235 & 19 \\
\hline Dexamethasone monotherapy & 0.057 & 0.068 & 0.059 & 0.089 & 0.0721 & 20 \\
\hline Oblimersen + dexamethasone* & 0.041 & 0.011 & NA & NA & 0.017 & 21 \\
\hline
\end{tabular}

Notes: A $20 \%, 35 \%$, and $45 \%$ weight was given to the NRR, PFS, and OS, respectively. *Since data on PFS and OS were not available, a $20 \%$ and $80 \%$ weight was assigned to NRR and TTP, respectively.

Abbreviations: NA, not available; NRR, non-response rate; TTP, time-to-progression; PFS, progression-free survival; OS, overall survival.

effective ones, with lenalidomide and dexamethasone as the backbone plus one of the four latest agents (daratumumab, ixazomib, carfilzomib, or elotumumab). The second subgroup includes 11 regimens $(52.4 \%$, weighted average from $\sim 0.4$ to $\sim 0.7$ ), which show moderate efficacy in RRMM patients and are mostly doublet or triplet regimens. The third subgroup includes six regimens $(28.6 \%$, weighted average from 0.0 to 0.25 ), which are mostly singlet or doublet regimens having limited efficacy.

An important advantage of NMA over traditional metaanalysis is the possibility of ranking multiple treatment options. The SUCRA score, derived from the probability that a given treatment has a certain rank and calculated from the posterior distributions of all treatments, is widely used for ranking treatments in Bayesian NMAs. In this study, we applied a weighted averaging strategy to rank the regimens for overall efficacy. We assigned weights to different outcome measures in accordance with their importance. The OS is the "gold standard" outcome measure for the evaluation of long-term anticancer efficacy, the PFS and TTP are widely acknowledged surrogates of OS, and the treatment response associated with early efficacy may differ from long-term outcomes. Therefore, we gave a $20 \%, 35 \%$, and $45 \%$ weight to NRR, PFS/TTP, and
OS, respectively. We did not take safety into account, because to date no adequate data were available to include all treatment options in one network for any single adverse event outcome. A systematic review less quantitatively intense may be a good choice for further investigation on safety profiles. We do not recommend any ranking based on a combined quantitative analysis of efficacy and safety, because they are two facets distinct from one another, and it is very difficult to assign a rational and appropriate weight to each outcome.

The present study, similar to its preceding works, also has some limitations. First, although the relative importance of different outcome measures was considered, the appropriateness of the arbitrary assignment of weights needs further verification. Besides, it should acknowledge the limitations of the NMA fully when comparing benefits from the experimental treatments in patient populations that differ in previous treatments, cytogenetic risks, subsequent stem-cell transplantation, maintenance therapy, and so on. Although it could be possible to make adjustment by using approaches such as network meta-regression, the immaturity of methodology, limited number of included trials and frequent missingness in certain study-level variables make it difficult and less reliable. 


\section{Conclusion}

In conclusion, the combination of daratumumab, lenalidomide, and dexamethasone may currently be the most effective regimen in the population of RRMM patients. Triplet regimens containing daratumumab, ixazomib, carfilzomib, or elotumumab plus lenalidomide and dexamethasone can be recommended as the first-line therapies for RRMM patients.

\section{Disclosure}

The authors report no conflicts of interest in this work.

\section{References}

1. van Beurden-Tan CHY, Franken MG, Blommestein HM, Uyl-de Groot CA, Sonneveld P. Systematic literature review and network metaanalysis of treatment outcomes in relapsed and/or refractory multiple myeloma. J Clin Oncol. 2017;35(12):1312-1319.

2. Botta DCC, Ciliberto D, Rossi M, et al. Network meta-analysis of randomized trials in multiple myeloma: efficacy and safety in relapsed/ refractory patients. Blood Adv. 2017;1(7):455-466.

3. Salanti G, Ades AE, Ioannidis JP. Graphical methods and numerical summaries for presenting results from multiple-treatment meta-analysis: an overview and tutorial. J Clin Epidemiol. 2011;64(2):163-171.

4. San Miguel J, Weisel K, Moreau P, et al. Pomalidomide plus low-dose dexamethasone versus high-dose dexamethasone alone for patients with relapsed and refractory multiple myeloma (MM-003): a randomised, open-label, phase 3 trial. Lancet Oncol. 2013;14(11):1055-1066.

5. Richardson PG, Siegel DS, Vij R, et al. Pomalidomide alone or in combination with low-dose dexamethasone in relapsed and refractory multiple myeloma: a randomized phase 2 study. Blood. 2014;123(12):1826-1832.

6. Morgan G, Palumbo A, Dhanasiri S, et al. Overall survival of relapsed and refractory multiple myeloma patients after adjusting for crossover in the MM-003 trial for pomalidomide plus low-dose dexamethasone. Br J Haematol. 2015;168(6):820-823.

7. San-Miguel JF, Hungria VT, Yoon SS, et al. Overall survival of patients with relapsed multiple myeloma treated with panobinostat or placebo plus bortezomib and dexamethasone (the PANORAMA 1 trial): a randomised, placebo-controlled, phase 3 trial. Lancet Haematol. 2016;3(11):e506-e515.

8. Dimopoulos MA, Lonial S, White D, et al. Elotuzumab plus lenalidomide/dexamethasone for relapsed or refractory multiple myeloma: ELOQUENT-2 follow-up and post-hoc analyses on progression-free survival and tumour growth. Br J Haematol. 2017;178(6):896-905.

9. Hajek R, Masszi T, Petrucci MT, et al. A randomized phase III study of carfilzomib vs low-dose corticosteroids with optional cyclophosphamide in relapsed and refractory multiple myeloma (FOCUS). Leukemia. 2017;31(1):107-114.

10. Hou J, Jin J, Xu Y, et al. Randomized, double-blind, placebo-controlled phase III study of ixazomib plus lenalidomide-dexamethasone in patients with relapsed/refractory multiple myeloma: China Continuation study. J Hematol Oncol. 2017;10(1):137.

11. Richardson PG, Sonneveld P, Schuster MW, et al. Bortezomib or highdose dexamethasone for relapsed multiple myeloma. $N$ Engl $J$ Med. 2005;352(24):2487-2498.

12. Richardson PG, Sonneveld P, Schuster M, et al. Extended follow-up of a phase 3 trial in relapsed multiple myeloma: final time-to-event results of the APEX trial. Blood. 2007;110(10):3557-3560.

13. Dimopoulos M, Spencer A, Attal M, et al. Lenalidomide plus dexamethasone for relapsed or refractory multiple myeloma. $\mathrm{NEngl} \mathrm{J} \mathrm{Med.}$ 2007;357(21):2123-2132.

14. Orlowski RZ, Nagler A, Sonneveld P, et al. Randomized phase III study of pegylated liposomal doxorubicin plus bortezomib compared with bortezomib alone in relapsed or refractory multiple myeloma: combination therapy improves time to progression. J Clin Oncol. 2007;25(25):3892-3901.
15. Weber DM, Chen C, Niesvizky R, et al. Lenalidomide plus dexamethasone for relapsed multiple myeloma in North America. $N$ Engl J Med. 2007;357(21):2133-2142.

16. Chanan-Khan AA, Niesvizky R, Hohl RJ, et al. Phase III randomised study of dexamethasone with or without oblimersen sodium for patients with advanced multiple myeloma. Leuk Lymphoma. 2009;50(4): 559-565.

17. Dimopoulos MA, Chen C, Spencer A, et al. Long-term follow-up on overall survival from the MM-009 and MM-010 phase III trials of lenalidomide plus dexamethasone in patients with relapsed or refractory multiple myeloma. Leukemia. 2009;23(11):2147-2152.

18. Garderet L, Iacobelli S, Moreau P, et al. Superiority of the triple combination of bortezomib-thalidomide-dexamethasone over the dual combination of thalidomide-dexamethasone in patients with multiple myeloma progressing or relapsing after autologous transplantation: the MMVAR/IFM 2005-04 Randomized Phase III Trial from the Chronic Leukemia Working Party of the European Group for Blood and Marrow Transplantation. J Clin Oncol. 2012;30(20):2475-2482.

19. Hjorth M, Hjertner O, Knudsen LM, et al. Thalidomide and dexamethasone vs. bortezomib and dexamethasone for melphalan refractory myeloma: a randomized study. Eur J Haematol. 2012;88(6):485-496.

20. Kropff M, Baylon HG, Hillengass J, et al. Thalidomide versus dexamethasone for the treatment of relapsed and/or refractory multiple myeloma: results from OPTIMUM, a randomized trial. Haematologica. 2012;97(5):784-791.

21. Dimopoulos M, Siegel DS, Lonial S, et al. Vorinostat or placebo in combination with bortezomib in patients with multiple myeloma (VANTAGE 088): a multicentre, randomised, double-blind study. Lancet Oncol. 2013;14(11):1129-1140.

22. White D, Kassim A, Bhaskar B, Yi J, Wamstad K, Paton VE. Results from AMBER, a randomized phase 2 study of bevacizumab and bortezomib versus bortezomib in relapsed or refractory multiple myeloma. Cancer. 2013;119(2):339-347.

23. San-Miguel JF, Hungria VT, Yoon SS, et al. Panobinostat plus bortezomib and dexamethasone versus placebo plus bortezomib and dexamethasone in patients with relapsed or relapsed and refractory multiple myeloma: a multicentre, randomised, double-blind phase 3 trial. Lancet Oncol. 2014;15(11):1195-1206.

24. Lonial S, Dimopoulos M, Palumbo A, et al. Elotuzumab therapy for relapsed or refractory multiple myeloma. $N$ Engl J Med. 2015;373(7): 621-631.

25. Orlowski RZ, Gercheva L, Williams C, et al. A phase 2, randomized, double-blind, placebo-controlled study of siltuximab (anti-IL-6 mAb) and bortezomib versus bortezomib alone in patients with relapsed or refractory multiple myeloma. Am J Heamtol. 2015;90(1):42-49.

26. Stewart AK, Rajkumar SV, Dimopoulos MA, et al. Carfilzomib, lenalidomide, and dexamethasone for relapsed multiple myeloma. $N$ Engl J Med. 2015;372(2):142-152.

27. Dimopoulos MA, Oriol A, Nahi H, et al. Daratumumab, lenalidomide, and dexamethasone for multiple myeloma. NEngl J Med.2016;375(14): 1319-1331.

28. Dimopoulos MA, Moreau P, Palumbo A, et al. Carfilzomib and dexamethasone versus bortezomib and dexamethasone for patients with relapsed or refractory multiple myeloma (ENDEAVOR): a randomised, phase 3, open-label, multicentre study. Lancet Oncol. 2016;17(1):27-38.

29. Jakubowiak A, Offidani M, Pegourie B, et al. Randomized phase 2 study: elotuzumab plus bortezomib/dexamethasone vs bortezomib/dexamethasone for relapsed/refractory MM. Blood. 2016;127(23):2833-2840.

30. Moreau P, Masszi T, Grzasko N, et al. Oral ixazomib, lenalidomide, and dexamethasone for multiple myeloma. $N$ Engl $J$ Med. 2016;374(17):1621-1634.

31. Orlowski RZ, Nagler A, Sonneveld P, et al. Final overall survival results of a randomized trial comparing bortezomib plus pegylated liposomal doxorubicin with bortezomib alone in patients with relapsed or refractory multiple myeloma. Cancer. 2016;122(13):2050-2056.

32. Palumbo A, Chanan-Khan A, Weisel K, et al. Daratumumab, bortezomib, and dexamethasone for multiple myeloma. $N$ Engl $J$ Med. 2016;375(8):754-766. 
Cancer Management and Research

\section{Publish your work in this journal}

Cancer Management and Research is an international, peer-reviewed open access journal focusing on cancer research and the optimal use of preventative and integrated treatment interventions to achieve improved outcomes, enhanced survival and quality of life for the cancer patient

The manuscript management system is completely online and includes

Submit your manuscript here: https://www.dovepress.com/cancer-management-and-research-journal

a very quick and fair peer-review system, which is all easy to use. Visit $\mathrm{http}: / / \mathrm{www}$.dovepress.com/testimonials.php to read real quotes from published authors. 